\title{
Facilitating scientific discovery through crowdsourcing and distributed participation
}

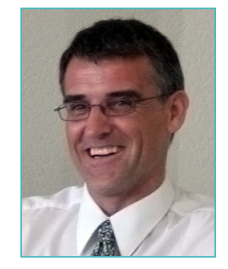

\section{Antony Williams}

Royal Society of Chemistry
Antony Williams is the Vice President, Strategic Development at the Royal Society of Chemistry and leads their eScience efforts. He was one of the founders for Chemspider, one of the world's primary internet resources for chemists now hosted by RSC and providing access to over 28 million unique chemicals linked out to over 400 data sources on the internet. He was the Chief Science Officer for Advanced Chemistry Development (ACD/Labs) focusing his cheminformatics skills on structure representation, nomenclature and analytical data handling. He is an NMR spectroscopist by training with a PhD from the University of London and is an accomplished author with over 140 publications, and many book chapters or books. He is known as the ChemConnector in the chemistry social network and is passionate about providing tools for data sharing, collaboration and alternative metrics for recognizing the contributions of scientists.

Science has evolved from the isolated individual tinkering in the lab, through the era of the "gentleman scientist" with his or her assistant(s), to group-based then expansive collaboration and now to an opportunity to collaborate with the world. With the advent of the internet the opportunity for crowd-sourced contribution and large-scale collaboration has exploded and, as a result, scientific discovery has been further enabled. The contributions of enormous open data sets, liberal licensing policies and innovative technologies for mining and linking these data has given rise to platforms that are beginning to deliver on the promise of semantic technologies and nanopublications, facilitated by the unprecedented computational resources available today, especially the increasing capabilities of handheld devices. The speaker will provide an overview of his experiences in developing a crowdsourced platform for chemists allowing for data deposition, annotation and validation. The challenges of mapping chemical and pharmacological data, especially in regards to data quality, will be discussed. The promise of distributed participation in data analysis is already in place. 